\title{
Análise da eficácia do taping patelar associado a um programa de tratamento fisioterapêutico em indivíduos do sexo feminino com disfunção patelofemoral Evaluation of patellar taping efficacy associated to physical therapy in women with patellofemoral dysfunction
}

\author{
Rafael Aleixo Favarini, Ft.*, Lygia Paccini Lustosa, M.Sc.*
}

*Unicentro Newton Paiva, Belo Horizonte MG

\section{Resumo}

A síndrome dolorosa patelofemoral é descrita como uma disfunção multifatorial, de difícil tratamento. No programa de reabilitação são propostas várias terapêuticas que visam o fortalecimento do mecanismo extensor do joelho no intuito de promover melhor a estabilidade para a articulação. Um dos recursos utilizados é o taping de McConnell associado ao programa de exercícios. O objetivo do estudo foi verificar a eficácia do uso do taping patelar de $M c$ Connell na diminuição da dor e na melhora do desempenho funcional, em um programa de tratamento, de indivíduos do sexo feminino, com diagnóstico de SDPF. Participaram do estudo cinco indivíduos, do sexo feminino, com idade média de 21,5 anos $( \pm 2,57)$. Todas as voluntárias foram avaliadas pelo mesmo examinador antes, após a $9^{a}$. sessão de tratamento e após a $18^{\mathrm{a}}$. sessão. A avaliaçáo constou de testes de flexibilidade, testes específicos para diagnóstico de SDPF, Escala Visual Analógica de Dor para quantificar o nível de dor e aplicaçáo da Escala de Karlsson para quantificar o desempenho funcional. Três voluntárias realizaram tratamento através de alongamentos específicos e exercícios de reforço muscular em cadeia cinética aberta (Grupo I). Duas voluntárias realizaram os mesmos exercícios, mas com a colocação do taping patelar segundo $M c$ Connell, antes da realização dos exercícios de reforço muscular (Grupo II). Os resultados não demonstraram diferença significativa entre os grupos, quanto a melhora da dor e do desempenho funcional após a $9^{a}$. sessão $(\mathrm{p}$ $=0,34, p=0,08$, respectivamente). Também não houve diferença significativa entre os grupos após a $18^{\mathrm{a}} \cdot(\mathrm{p}>0,18)$. Não é possível afirmar, através dos resultados obtidos, que o uso do taping patelar associado ao programa de exercícios para o tratamento da SDPF, seja mais eficaz na melhora da dor e no desempenho funcional, que o uso somente do programa de exercícios.

Palavras-chave: taping patelar, disfunção patelofemoral, dor, desempenho funcional.

\begin{abstract}
Patellofemoral painful syndrome (PPS) is described as a hard to treat multifatorial dysfunction. Various conservative therapeutic approaches are proposed to manage such syndrome with the intention to strengthen the knee extensor mechanism to promote better joint stability. One of the rehabilitation resources is the $\mathrm{McC}$ Connell taping technique associated to physical therapy. The goal of this study was to verify the efficacy of patellar taping in reducing pain and improving functional performance associated to a treatment program in female patients diagnosed with PPS. Five female subjects, average age of $21,5( \pm 2,57)$ years, participated in the study. The same examiner, before and after the 9th and 18th treatment session, evaluated them. Evaluation was comprised of flexibility tests, specific tests to diagnose PPS, pain analogue visual scale to quantify the intensity of pain and Karlsson scale to quantify functional performance. Three volunteers were submitted to specific stretching and strengthening muscle exercises in open kinetic chain (group 1). Two volunteers performed the same exercises done by the group 1 plus patellar taping technique described by McConnell, before performing the strengthening exercises (group 2). Results showed no significant difference between groups concerning pain relief and functional performance after the $9^{\text {th }}$ session $(p=0,34, p=0,08$, respectively). Also, there was no significant statistical difference between both groups after the $18^{\text {th }}$ session $(p>0,18)$. It is not possible to affirm, through the present results, whether the patellar taping technique associated to physical therapy to treat PPS shows more efficacies to reduce pain and functional performance than exercises alone.
\end{abstract}

Key-words: taping patellar, patellofemoral disfunction, pain, functional outcomes. 


\section{I ntrodução}

O termo síndrome dolorosa patelofemoral (SDPF) define uma queixa comum de dor anterior ou retropatelar no joelho $[1,2]$. Essa disfunçáo está geralmente associada com o exercício e piora com atividades que aumentam as forças compressivas do joelho como o permanecer por longos períodos na posição sentada, o subir e descer escadas e o agachar [1-10]. Tem maior incidência em adolescentes do sexo feminino e em indivíduos ativos [1], com idade entre 10 e 35 anos $[3,7,8,11,12]$.

A SDPF é discutida como uma afecção multifatorial com causas diversas, mas talvez a mais comum seja a síndrome de deslocamento lateral [13]. Alguns dos fatores que podem desencadear o deslocamento lateral da patela incluem as anormalidades ósseas, a tensão do retináculo lateral e da banda iliotibial [4], a tensão do músculo isquiosural e/ou do músculo gastrocnêmio, o tendão patelar alongado, a patela alta, o mau alinhamento da tíbia $[2,14,15]$, o geno valgo, o aumento do ângulo Q, a anteversão femoral, o joelho recurvatum, a pronação excessiva do pé e os traumas [2,4,13-15].

O tratamento fisioterapêutico para a dor na articulação patelofemoral é direcionado principalmente para a reabilitação do músculo quadríceps e especificamente, o músculo vasto medial oblíquo (VMO), que é definido como o estabilizador dinâmico medial da patela $[16,17]$. Esse tratamento inclui geralmente o fortalecimento do músculo quadríceps favorecendo a ativaçáo do músculo $\mathrm{VMO}$, o alongamento do músculo isquiosural, do gastrocnêmio, do tensor da fáscia e da banda iliotibial, o treinamento da estabilidade articular, a aplicação do taping patelar ou o uso do bracing, a indicação de órteses para correção da pronação excessiva da subtalar e as modificaçôes das atividades de vida diária [2,3,5,11-14,1721]. Para a realização dos exercícios de fortalecimento Laprade et al. [7] e Fonseca et al. [22] avaliaram uma série de exercícios utilizados na prática clínica em cadeia cinética aberta e em cadeia cinética fechada, com o objetivo de demonstrar a sua eficácia através da análise da ativação muscular. Esses autores sugeriram que exercícios envolvendo a extensão do joelho associado à rotação da tíbia poderiam ser utilizados por apresentar uma melhor relação entre o músculo VMO e o músculo vasto lateral $[7,22]$.

McConnell [14] introduziu a técnica do taping patelar em seu programa de tratamento em meados dos anos 80, com o objetivo de melhorar o posicionamento da patela, diminuindo a dor e favorecendo o fortalecimento do músculo quadríceps. A autora preconiza que a correçáo da patela e o seu melhor posicionamento favorecem a função do músculo quadríceps.

O objetivo do estudo foi verificar a eficácia do uso do taping patelar de McConnell na diminuição da dor e na melhora do desempenho funcional, em um programa de tratamento, de indivíduos do sexo feminino com diagnóstico de SDPF.

\section{Material e métodos}

Foram convidados a participar do estudo indivíduos do sexo feminino, com idade variando entre 15 e 35 anos, que apresentavam características de SDPF. Os critérios de exclusão para o estudo foram história pregressa de cirurgia de joelho, instabilidade patelar, lesão meniscal, lesáo ligamentar e fraturas prévias dos membros inferiores. As voluntárias foram recrutadas através de cartazes afixados em uma instituição de ensino particular e assinaram um termo de livre consentimento e esclarecido, concordando em participar do estudo. A mesma avaliação foi repetida após a realização da nona sessão e ao final do tratamento ( $18^{\mathrm{a}}$. sessão), pelo mesmo examinador que não teve conhecimento em momento algum, do grupo de tratamento das participantes. Após a avaliação inicial, as voluntárias foram sorteadas para o início do tratamento para Grupo I ou Grupo II. O Grupo I realizou exercícios de alongamento específicos conforme a avaliação e exercícios para reforço do músculo quadríceps em cadeia cinética aberta. O Grupo II realizou os mesmos exercícios que o Grupo I, mas associado ao uso do taping patelar, conforme a técnica descrita por McConnell [14], colocado pelo mesmo examinador, antes do início dos exercícios de reforço muscular. Esse examinador acompanhou todo o tratamento, mas não participou das avaliaçôes.

Dessa forma, foram avaliadas 13 mulheres, com idade variando entre 15 e 25 anos ( $21,5 \pm 2,57$ anos). Dessas voluntárias, quatro não iniciaram o tratamento. Das nove participantes que deram início ao tratamento, uma abandonou o tratamento após a primeira sessão, duas abandonaram o tratamento após a segunda sessão e uma após a quarta sessão. Todas elas alegaram motivos pessoais e dificuldade quanto ao horário e deslocamento para o local de tratamento. Apenas cinco participantes deram continuidade ao estudo, concluindo o tratamento. O Grupo I constou entáo de três voluntárias e o Grupo II de duas voluntárias.

A avaliação fisioterapêutica constou da aplicação da escala de Karlsson [23], específica para a articulação patelofemoral. Essa escala foi preenchida pela própria participante, tendo recebido apenas a informação que os sinais e sintomas referiam-se a "última semana" antes da aplicação da mesma. Todas as voluntárias foram orientadas a manter o mesmo nível de atividade física durante a realização do tratamento, sem dar início a nenhuma atividade nova, para que náo houvesse possíveis alteraçóes em relação ao quadro clínico e do desempenho funcional. Para quantificar o nível de dor utilizou-se a Escala Visual Analógica de Dor [2]. Os testes para pesquisa da flexibilidade muscular foram o teste de Ober para o encurtamento do músculo tensor da fáscia lata [24], o teste de Ely para o encurtamento do músculo reto femoral [24], o teste de Thomas modificado para avaliar a presença de encurtamento do músculo iliopsoas [24], o teste para encurtamento de adutores [25] e o teste para encurtamento dos isquiosurais [25]. Finalmente, foram realizados testes especiais 
para a disfunção patelofemoral: teste de McConnell, sinal de Zohler e sinal de Frund [24]. Essa mesma avaliação foi repetida pelo mesmo examinador, que náo teve conhecimento dos grupos de tratamento, após a voluntária realizar a $9^{a}$ sessão e após a $18^{\mathrm{a}}$ sessão de tratamento.

Durante o tratamento, tanto as participantes do grupo I quanto as do grupo II realizaram os mesmos exercícios de reforço muscular para os músculos extensores do joelho. Esses exercícios seguiram a descrição de Fonseca et al. [22] para a extensão do joelho em cadeia cinética aberta mantendo a rotação externa de quadril (Figura 1). Foram realizados ainda, os exercícios para a extensáo do joelho em cadeia cinética aberta direcionando o movimento para a rotação interna da tíbia e exercício para a extensão de joelho em posição neutra de acordo com a descriçáo de Laprade et al. (Figura 2 e 3) [7]. $\mathrm{O}$ tratamento foi realizado três vezes por semana, durante o período de seis semanas (total de 18 sessôes). Nas voluntárias do grupo II foi colocado o taping patelar (Figura 4) antes do início dos exercícios de reforço muscular citados acima.

Figura 1 - Contração isométrica em cadeia cinética aberta mantendo a rotação externa do quadril.

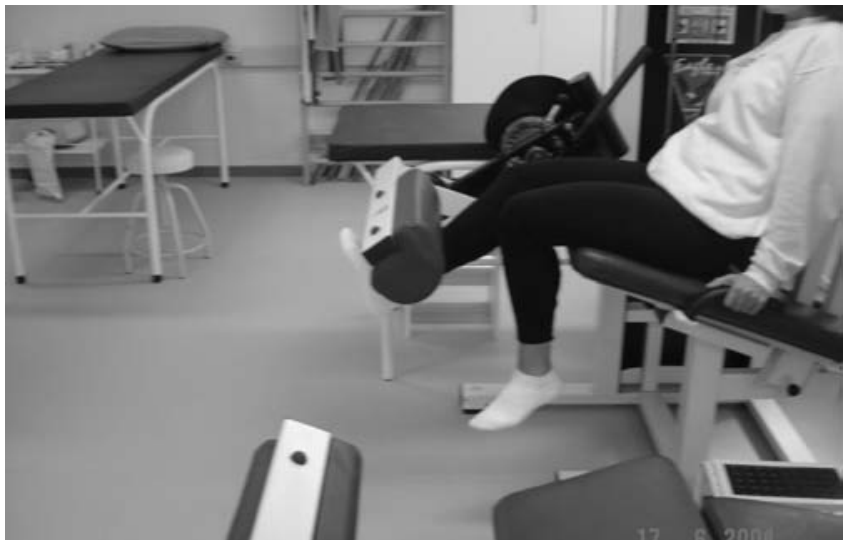

\section{Análise estatística}

Figura 2 - Contração isométrica em cadeia cinética aberta saindo da rotaçáo externa e direcionando para a rotaçâo interna do quadril.

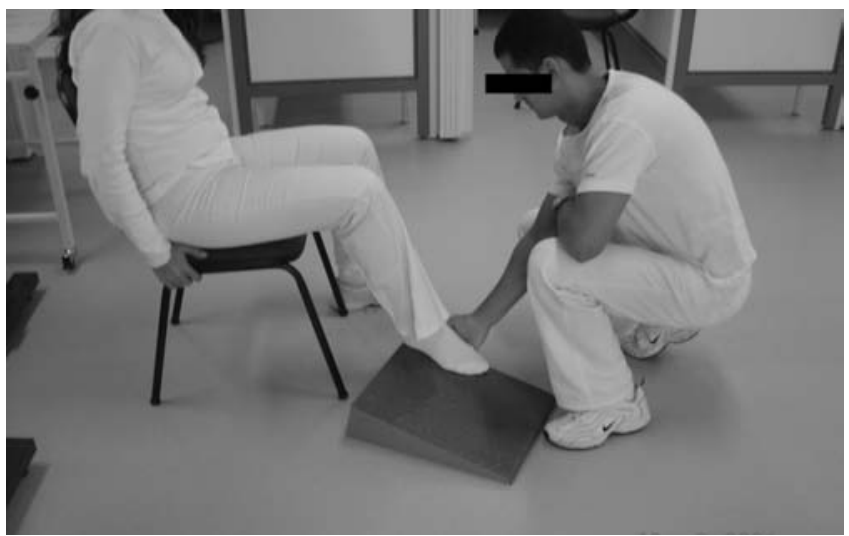

Figura 3 - Contração isométrica em cadeia cinética aberta com o membro inferior em posição neutral.

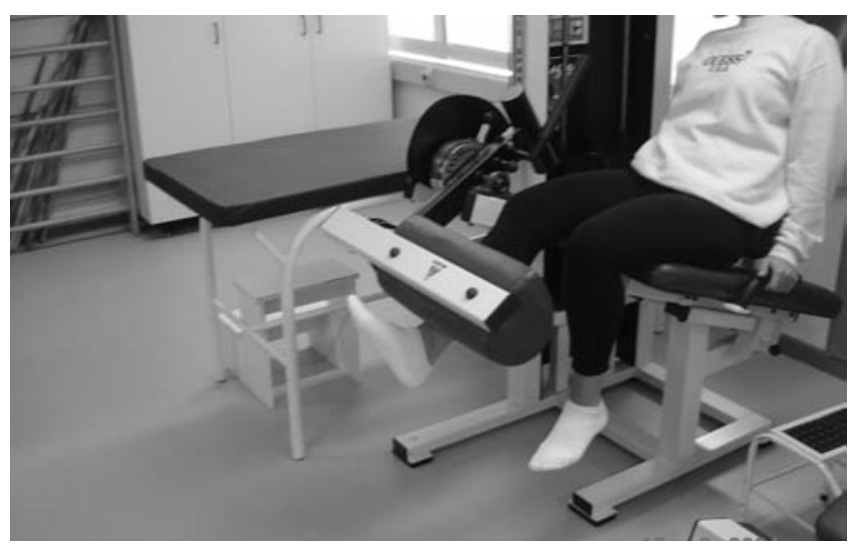

Figura 4 - Técnica de colocação do taping patelar segundo $\mathrm{Mc}$ Connell.
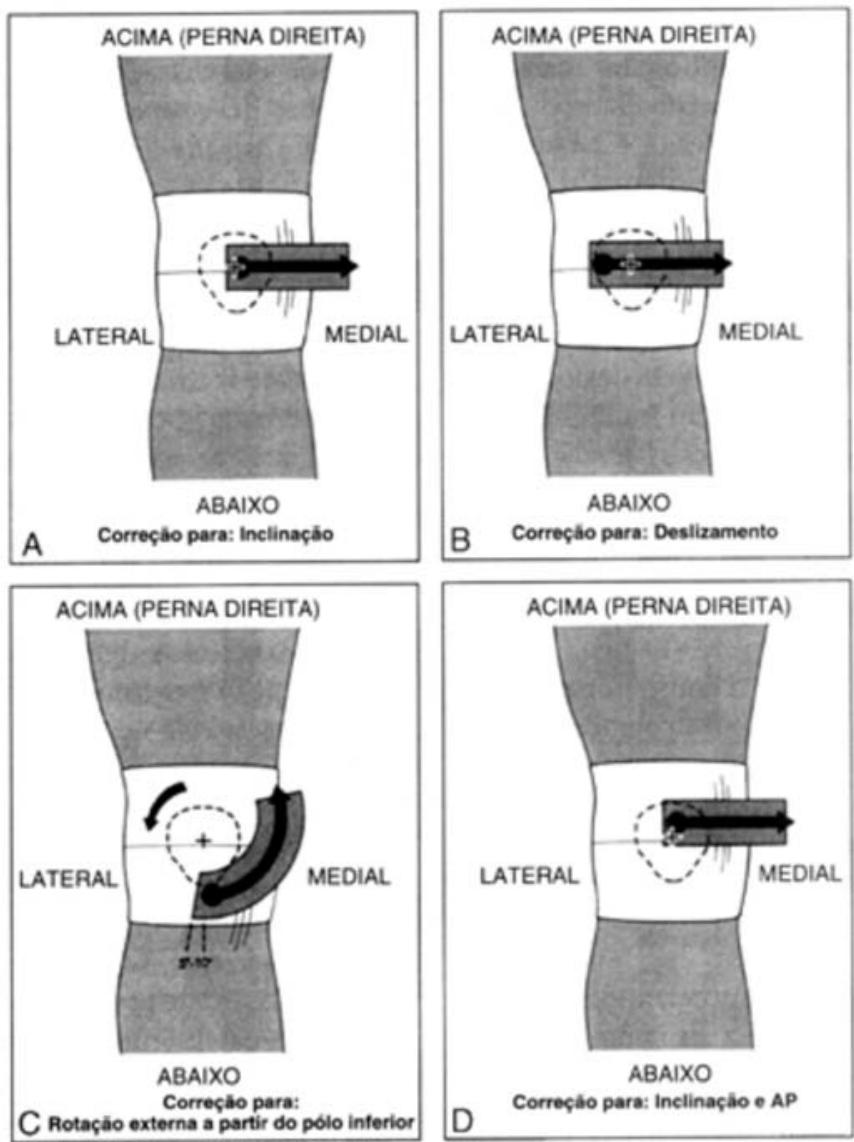

A análise estatística foi realizada através do programa estatístico SPSS 11.0. Para a comparação entre os grupos, quanto ao nível de dor e desempenho funcional, após a nona sessão e após a décima oitava sessão foi utilizando o teste não-paramétrico de Wilcoxon. O nível de significância foi estabelecido em $\alpha=0,05$. 


\section{Resultados}

Apesar de haver melhora em todos os parâmetros analisados, os resultados não demonstraram diferença significativa quando comparado os grupos I e II, quanto ao nível funcional $\mathrm{p}=0,08$ e quanto a melhora do nível da dor $\mathrm{p}=0,34$, após a nona sessão de tratamento. Da mesma forma quando comparados os grupos após a $18^{a}$. sessão, também não houve diferença significativa na melhora da funcionalidade e na melhora da dor $(\mathrm{p}>0,18)$.

\section{Discussão}

O presente estudo teve como objetivo verificar a eficácia da utilizaçáo do taping patelar associado a um programa de exercícios no tratamento de indivíduos com SDFP, quanto à melhora da dor e do desempenho funcional. No entanto, os resultados não demonstraram diferença significativa entre os grupos estudados, o que não permite afirmar que $o$ taping patelar aumente a eficácia do tratamento da SDFP. $\mathrm{O}$ tratamento das disfunçôes patelofemorais baseia-se no fortalecimento do músculo VMO, no intuito de estabelecer uma maior estabilidade articular $[14,26]$. Acredita-se que esse fortalecimento muscular possa promover uma maior medializaçáo da patela, levando ao seu melhor alinhamento durante a extensão do joelho [14]. No entanto, existem controvérsias quanto ao melhor exercício e a melhor ativação desse grupo muscular [26]. O taping patelar proposto por McConell teve como objetivo não só a melhora da dor, mas também uma maior possibilidade do posicionamento adequado da patela. Bockrath et al. [17], utilizando o taping patelar durante o tratamento da SDFP, demonstraram que houve significativa redução no nível da dor, comprovando sua eficácia no resultado do tratamento. Ao contrário, Kowall et al. [12] também comparando grupos que utilizaram o taping com aqueles que náo utilizaram, demonstraram que não houve diferença significativa entre os grupos estudados, o que corrobora com os resultados encontrados neste estudo. No presente estudo, uma das limitaçôes que pode ter interferido nos resultados foi o número reduzido de participantes envolvidos, tornando a amostra muito pequena.

Com relação ao tempo de tratamento, foi estabelecido o período de 6 semanas baseado no estudo de Crossley et al. [2] que demonstrou ser necessárias 6 semanas de tratamento para o alívio da dor utilizando o taping patelar associado a um programa de exercícios. No entanto, o período ideal de tratamento também apresenta controvérsias, visto existirem evidências que 4 semanas podem ser suficientes para a melhora da dor e retorno completo à funcionalidade, como afirmam Wittingham et al. [27]. Os resultados observados no presente estudo demonstraram melhora nos dois grupos principalmente entre a $1^{\text {a }}$. e $9^{\mathrm{a}}$. sessão de tratamento. Esses resultados foram semelhantes ao do estudo de McConnell [14] que encontrou que $92 \%$ dos pacientes tratados, ficaram livres da dor após 8 sessóes de tratamento utilizando o taping patelar e exercícios.

Apesar da ausência de diferença significativa entre os grupos estudados, pôde-se observar uma melhora dos sintomas e da função após o tratamento. Essa observação sugere que o tratamento apresenta resultados satisfatórios, mas que novos estudos deverão ser realizados, para verificar o real papel do taping patelar no tratamento da SDPF.

\section{Conclusão}

Apesar da melhora observada nos grupos estudados, os resultados encontrados neste estudo não permitem afirmar que a utilização do taping patelar associado a um programa de fortalecimento muscular, para o tratamento da SDPF, seja mais eficaz do que apenas o programa de exercícios, na diminuição da dor e na melhora do desempenho funcional.

\section{Referências}

1. Andrade PH, et al. Comparação da atividade elétrica dos músculos vasto medial oblíquo e vasto lateral oblíquo em indivíduos com disfunção fêmoro-patelar. Rev Fisioter Univ São Paulo 2001;8:65-71.

2. Crossley K, et al. Physical therapy for patellofemoral pain: a randomized, double-blinded, placebo-controlled trial. Am J Sports Med 2002; 30:857-65.

3. Cowan SM, et al. Physical therapy alters recruitment of the vasti in patellofemoral pain syndrome. Med Sci Sports Exerc 2002;34:1879-85.

4. Cowan SM, Bennell KL, Hodges PW. Therapeutic patellar taping changes the timing of vasti muscle activation in people with patellofemoral pain syndrome. Clin J Sport Med 2002;12:33947.

5. Crossley K, et al. Patellar taping: is clinical success supported by scientific evidence? Man Ther 2000;5:142-50.

6. Handfield T, Kramer J. Effect of McConnell taping on perceived pain and knee extensor torque during isokinetic exercise performed by patients with patellofemoral pain syndrome. Physiother Can 2000;2:39-44.

7. Laprade J, Culham E, Brouwer B. Comparison of five isometric exercises in the recruitment of the vastus medialis oblique in persons with or without patellofemoral pain syndrome. J Orthop Sports Phys Ther 1998;27:197-204.

8. Muller K, Snyder-Mackler L. Diagnosis of patellofemoral pain after arthroscopic meniscectomy. J Orthop Sports Phys Ther 2000;30:138-42.

9. Powers $\mathrm{CM}$ et al. The effects of patellar taping on stride characteristics and joint motion in subjects with patellofemoral pain. J Orthop Sports Phys Ther 1997;26: 286-91.

10. Salsich GB et al. The effects of patellar taping on knee kinetics, kinematics, and vastus lateralis muscle activity during stair ambulation with patellofemoral pain. J Orthop Sports Phys Ther 2002;32:3-10.

11. Ernst GP, Kawaguchi J, Saliba E. Effect of patellar taping on knee kinetics of patients with patellofemoral pain syndrome. J Orthop Sports Phys Ther 1999; 29:661-7. 
12. Kowall MG, et al. Patellar taping in the treatment of patellofemoral pain. A prospective randomized study. Am J Sports Med 1996;24:61-6.

13. Larsen B, et al. Patellar taping: a radiographic examination of the medial glide technique. Am J Sports Med 1995;23:465-71.

14. McConnell J. The management of chondromalacia patellae: A long-term solution. Aust J Physiother 1986;32:215-223.

15. Powers CM, et al. Criterion-related validity of a clinical measurement to determine the medial/lateral component of patellar orientation. J Orthop Sports Phys Ther 1999;29:372-7.

16. Holmes SW, Clancy WG. Clinical classification of patellofemoral pain and dysfunction. J Orthop Sports Phys Ther 1998;28:299-306.

17. Bockrath K, et al. Effects of patella taping on patella position and perceived pain. Med Sci Sports Exerc 1993;25:989-92.

18. Watson CJ, et al. Reliability of McConnell's classification of patellar orientation in symptomatic and asymptomatic subjects. J Orthop Sports Phys Ther 1999,29:378-385.

19. Herrington LC. The inter-tester reliability of a clinical measurement used to determine the medial-lateral orientation of the patella. Man Ther 2002;7:163-7.
20. Fulkerson JP. Diagnosis and treatment of patients with patellofemoral pain. Am J Sports Med 2002;30:447-56.

21. Gigante A et al. The effects of patellar taping on incongruent. A computed tomography study. Am J Sports Med 200;29:88-92.

22. Fonseca ST et al. Análise eletromiográfica dos músculos vasto medial oblíquo e vasto lateral em exercícios usados no tratamento da síndrome da dor patelofemoral. Rev Fisioter Univ São Paulo 2001;8:1-10.

23. Karlsson J, Thomeé R, Sward L. Eleven year follow-up of patellofemoral pain syndrome. Clinical J Sport Med 1996;6:22-6.

24. Magee DJ. Quadril. In: Avaliação músculo esquelética. $3^{\mathrm{a}}$ ed. São Paulo: Manole; 2002. p.587-8.

25. Alter MJ. Ciência da flexibilidade. 2a ed. Porto Alegre: Artmed; 1999. p.304-308.

26. Serrão FV, Nunes CM, Bérzin F, Candolo C, Monteiro-Pedro V. Effect of tibia rotation on the electromyographical activity of the vastus medialis oblique and vastus lateralis longus muscles during isometric leg press. Physical therapy in Sport 2005;6:15-23.

27. Wittingham M, Palmer S, Macmillan F. Effects of taping on pain and function in patellofemoral pain syndrome: A randomized controlled trial. J Orthop Sports Phys Ther 2004;34:504-10. 